\title{
Lower Hemi-Continuity, Open Sections, and Convexity: Counter Examples in Infinite Dimensional Spaces
}

\author{
Adib Bagh \\ Economics and Mathematics Departments, University of Kentucky, Lexington, USA \\ Email: adib.bagh@uky.edu
}

Received March 5, 2012; revised March 26, 2012; accepted April 6, 2012

\begin{abstract}
A lower hemi-continuous correspondence with open and convex values in $R^{n}$ must have open lower sections. This wellknown fact has been used to establish the existence of continuous selections, maximal elements, and fixed points of correspondences in various economic applications. Since there is an increasing number of economic models that use correspondences in an infinite-dimensional setting, it is important to know whether or not the above fact remains valid in such applications. The aim of this paper is to show that the above fact no longer holds when $R^{n}$ is replaced with an infinite-dimensional space. This is accomplished by using the standard orthonormal base in a Hilbert space $H$ to construct two correspondences with values in $H$ equipped with the weak topology. The first correspondence is lower hemi-continuous with open and convex values but does not have open lower sections. The second is a lower hemi-continuous correspondence that fails to have an open graph despite having open and convex upper and lower sections. These counter-examples demonstrate that in an infinite-dimensional setting, it is no longer possible to rely on the geometric properties of a lower hemi-continuous map (the convexity of its sections) to establish the topological properties (open lower sections, open graph) needed in many economic applications.
\end{abstract}

Keywords: Lower Hemi-Continuity; Open Lower Sections; Open Graph; Value Function; Maximal Element; Nash Equilibrium; Weak Topology

\section{Introduction}

Correspondences with open lower sections play an important role in general equilibrium analysis and in game theory. In general, the lower sections of a lower hemicontinuous (lhc) correspondence need not be open. However, for an lhc correspondence with upper sections that are open and convex in $R^{n}$, we have the following result (Proposition 11.70 in [1], Theorem 5.9 in [2]):

Theorem 1. Let $S$ be a correspondence from a Hausdorff space $X$ to $R^{n}$. If $S$ is lower hemi-continuous with upper sections (values) that are convex and open in $R^{n}$, then $S$ has open lower sections.

When $S: R^{m} \mapsto R^{n}$ has convex lower and upper sections, the above theorem implies that $S$ is lhc with open upper sections, if and only if $S^{-1}$, the inverse of $S$, is lhc with open upper sections.

Theorem 1 has several applications in economics. For example, the statement "since $g\left(x_{0}\right) \in \operatorname{int} \Gamma\left(x_{0}\right)$ and $\Gamma$ is continuous, it follows that $g\left(x_{0}\right) \in \operatorname{int} \Gamma(x)$ in some neighborhood of $x_{0}$ " appears in the proof of a well-known theorem regarding the differentiability of the value function (Theorem 4.11, page 85 in [3]) (the set $\operatorname{int} \Gamma(x)$ denotes the interior of $\Gamma(x))$. The justifica- tion of this claim relies on Theorem 1; the correspondence $\Gamma$ has a range in $R^{n}$, and it is continuous with a convex graph. This implies that the correspondence $x \mapsto \operatorname{int} \Gamma(x)$ is lhc with open and convex upper sections, and the statement quoted from [3] follows immediately. ${ }^{1}$

For more on the application of Theorem 1 in establishing the differentiability of value functions, see [5] and Kim [6]. Theorem 1 has also been used to establish the existence of continuous selections, fixed points of best reply functions, and to establish the existence of equilibria for non-ordered preferences in abstract economies [7-10]. It is straightforward to show that Theorem 1 does not hold if the convexity requirement on the upper sections of $\mathrm{S}$ is dropped. It is also relatively easy to demonstrate that this theorem does not hold, if the upper sections of $\mathrm{S}$ are convex but are not open in $R^{n}$ (see page

\footnotetext{
${ }^{1}$ It is interesting to note that Aliprantis, Camera, and Ruscitti (page 454 in [4]) claimed that the statement we quoted from Theorem 4.11 in [3] was is not substantiated, which was one of the factors that motivated their search for an alternative approach for establishing the differentiability of value functions. Note also that when $\Gamma$ is continuous with convex sections, the conditions $y_{0} \in \operatorname{int} \Gamma\left(x_{0}\right)$ and $x_{0} \in \operatorname{int} \Gamma^{-1}\left(y_{0}\right)$, discussed at length in [4], are in fact equivalent.
} 
237 in [11] and page 9 in [12]). Keeping all the other assumptions the same, does the above theorem hold if $R^{n}$ is replaced with an arbitrary locally convex (infinitedimensional) space? (for examples of economic applications involving correspondences with values that are not in $R^{n}$ see [13-20]). As far as we know, there has not been a satisfactory answer to this question. In fact, in his book Mathematical Methods for Economists, Moore (page 273 in [1]) states "it may well be that Holly's proposition [Theorem 1] can be generalized to the extent of substituting an arbitrary locally convex Hausdorff space in place of $R^{n}$, although I am not sure whether or not this conjecture is correct". In this note, we show that the answer to our question is no, and that the above conjecture is incorrect.

\section{Two Counter-Examples}

Recall that a correspondence $S: X \mapsto Y$ is lhc at some point $x \in X$, if for every open set $V$ in $Y$ such that $V \cap S(x) \neq \phi$, there exists an open neighborhood $W$ of $x$ such that $V \cap S\left(x^{\prime}\right) \neq \phi$ for all $x^{\prime} \in W$ When $X$ is first countable, this definition is equivalent to the following: for any open set $V$ in $Y$ such $V \cap S(x) \neq \phi$, and for any sequence $x_{m} \rightarrow x$, there exists $m_{0}$ such that $V \cap S\left(x_{m}\right) \neq \phi$ for all $m \geq m_{0}$. Let $\ell^{2}$ be the space of square summable sequences in $R$. This is a separable Hilbert space with an inner product that we shall denote by $\langle\cdot, \cdot\rangle$. Let $\left\{e_{n}, n=1, \cdots\right\}$ be the standard orthonormal base in $\ell^{2}$ (the vector $e_{n}$ has 1 in the nth position and zeros everywhere else). Let $A$ be the collection $\bigcup_{n \geq 1}\left\{e_{n}\right\}$ and let $A_{0}=A \bigcup\{0\}$. Let $X$ be the unit ball $B_{1}(0)$ in $\ell^{2}$ equipped with the weak topology (i.e. $W$ is open in $X$, if and only if $W=W^{\prime} \cap B_{1}(0)$ for some set $W^{\prime}$ that is weakly open in $\ell^{2}$ ). Note that $A_{0}$ is the closure of $A$ in the weak topology of $\ell^{2}$. Therefore, $X \backslash A$ is not open in $X$ but $X \backslash A_{0}$ is. Finally, let $Y$ be the space $\ell^{2}$ equipped with the weak topology. The space $Y$ is an infinite-dimensional locally convex Hausdorff topological vector space. Since the unit ball in a separable reflexive Banach space is metrizable in the weak topology (Theorem 3.16 in [21]), $X$ is metrizable even though $Y$ is not.

Lemma 1. For every $e_{n} \in A$, let

$$
C_{n}=\left\{y \in Y \mid\left\langle e_{n}, y\right\rangle>1\right\} .
$$

The set $\cup_{n \geq 1} C_{n}$ is dense in $Y$.

Proof. For every $z \in Y$, let $z_{n}=z$ except for the nth component, which is set to be equal to 2 (anything bigger than 1 will work). For every $n \geq 1,\left\langle e_{n}, z_{n}\right\rangle=2$, and hence $z_{n} \in C_{n}$. Moreover, for every $x \in \ell^{2}$, $\lim _{n}\left\langle x, z_{n}\right\rangle=\langle x, z\rangle$, and therefore $z_{n}$ converges weakly to $z$.

Lemma 2. Let $c_{n}$ be defined as in Lemma 1. Let
$S: X \mapsto Y$ be a correspondence defined as follows:

$$
S(x)= \begin{cases}C_{n} & \text { when } x=e_{n} \\ Y & \text { otherwise. }\end{cases}
$$

Then, $S$ is lower hemi-continuous with upper sections that are open and convex in $Y$.

Proof. By the definition of the weak topology, for every $x \in \ell^{2}$, the set $\{y \in Y \mid\langle x, y\rangle>1\}$ is open in the weak topology. Hence, it is clear that for any $x \in \ell^{2}$, $S(x)$ is convex and open in $Y$. Let $x \in X \backslash A_{0}$ and let $V$ be an open set in $Y$ such that $V \cap S(x) \neq \phi$. Since $X \backslash A_{0}$ is open in $X$, there exists a neighborhood $W$ of $x$ such that $W \subset X \backslash A_{0}$ and $V \cap S\left(x^{\prime}\right)=V \cap S(x) \neq \phi$, for all $x^{\prime} \in W$. Hence, $S$ is lhc at $x$ Now let $e_{n_{0}}$ be some element in $A$, and let $x_{n} \rightarrow e_{n_{0}}$ in $X$, and without loss of generality assume that $X_{n}$ is not the constant sequence $e_{n_{0}}$ There exists $m_{0}$ such that for all $m \geq m_{0}$, $x_{m} \subset X \backslash A_{0}$. Otherwise, we can obtain a subsequence $e_{n_{k}}$ of $e_{n}$ such that $e_{n_{k}} \rightarrow e_{n_{0}} \quad$ weakly, contradicting the fact that $e_{n}$ has a unique weak limit (the sequence 0 ) given the fact that $X$ is Hausdorff. Now this implies that $S\left(x_{m}\right)=Y$ for all $m \geq m_{0}$. Hence, $S$ is lhc at $e_{n_{0}}$, and therefore it is lhc at any point in $A$. We still need to show that $S$ is lhc at $x=0$. Assume $S$ is not lhc at zero. Then, there exists an open set $V$ in $Y$ such that $V \cap S(0)=V \neq \phi$ and there exists $x_{n} \rightarrow 0$ in $X$ such that $V \cap S\left(x_{n}\right)=\phi$ for all $n$. This sequence has to be a subset of $A$ (i.e. a subsequence of $\left.e_{n}\right)$ since $S(x)=Y$ for any $x \in X \backslash A$. Without loss of generality, simply assume $x_{n}=e_{n}$ for all $n$. Let $z \in V$. By Lemma 1, there exists a sequence $z_{n} \in S\left(e_{n}\right)$ such $z_{n}$ converges weakly to $z$, and therefore $V \cap S\left(e_{n}\right) \neq \phi$ for some $n$, a contradiction. Hence, $S$ is lhc at zero.

Proposition 1. Let $S$ be defined as in Lemma 2. Then, $S$ is lower hemi-continuous with upper sections that are open and in convex in $Y$, and yet $S$ does not have open lower sections.

Proof. By Lemma 2, $S$ is lower hemi-continuous with upper sections that are open and in convex in $Y$. Moreover, $S^{-1}(0)=X \backslash A$, which is not an open set in $X$.

The closed graph theorem for correspondences asserts that a closed-valued correspondence with a compact range is upper hemi-continuous, if and only if it has a closed graph (Proposition 17.11 in [22]). This result, particularly when combined with Kakutani's fixed point theorem, has important applications in economics. It is then natural to ask if there exists an "open graph" theorem, i.e. a theorem asserting that a lower hemi-continuous correspondence with open and convex values is lower hemi-continuous, if and only if it has an open graph. ${ }^{2}$ The fact that this statement does in fact hold when the range of $S$ is $R^{n}$ was proved Zhou who used this

${ }^{2}$ The question regarding the existence of an "open graph” theorem was raised first by Bergstrom, Park, and Rader [23]. 
result to establish the existence of equilbria in various qualitative games.

Theorem 2. (Proposition 2 in [24]). Let $S$ be a correspondence from a Hausdorff space $X$ to $R^{n}$. If $S$ is lower hemi-continuous with open and convex upper sections in $R^{n}$, then $S$ has an open graph.

The setting of Proposition 1 can be slightly modified to show that when $S$ has an infinite dimensional range, Theorem 2 may not hold, even under the additional assumption that $S$ has open lower sections (Bergstrom, Parks, and Rader in [23] provided an example of a correspondence with open upper and lower sections but whose graph was is not open, In their example, however, the correspondence has non-convex upper section).

Proposition 2. Let $X$ and $Y$ be defined as before. Let $S: X \mapsto Y$ be defined by

$$
S(x)=\{y \in Y \mid\langle x, y\rangle<1\} .
$$

Then $S$ is lhe with open and convex upper and lower sections in $R^{n}$, yet the graph of $S$ is not open.

Proof. Clearly, $S$ has open and convex upper and lower sections (in $X$ and $Y$ respectively). Assume $S$ has an open graph in $X \times Y$, which means that set

$$
\text { gph } S=\{(x, y) \in X \times Y \mid\langle x, y\rangle<1\} .
$$

is open in $X \times Y$. This implies that the set

$$
C=\{(x, y) \in X \times Y \mid\langle x, y\rangle \geq 1\} .
$$

is closed in $X \times Y$, The sequence $\left\{\left(e_{n}, e_{n}\right)\right\}$ is contained in $C$, yet $(0,0)$, the limit of this sequence in the product topology on $X \times Y$, is not. This contradicts the fact that $C$ is closed. Therefore the graph of $S$ is not open.

Similar counter-examples based on Propositions 1 and 2 can be constructed if $\ell^{2}$ is replaced with any Hilbert space $H$ and $Y$ is taken to be $H$ equipped with the weak topology (for economic applications where the underlying space is equipped with the weak topology, see [13] and [18]).

\section{Conclusions}

For a lower hemi-continuous correspondence $S$ with values in a finite dimensional Euclidean space, the convexity of the upper and lower sections has very strong topological implications (Theorems 1 and 2) that can used to obtain existence results for maximal elements, fixed points, continuous selections, and Nash equilibria. However, there is an increasing number of economic applications that involve correspondences with values in infinite-dimensional spaces. Some of these applications consist of dynamic choice models over an infinite horizon [14]. Other applications consist of general equilibrium models that allow for infinite variation within the com- modities of the economy. This includes variations in the physical attributes of the goods, time of delivery, and the state of the world when delivery takes place $[12,13$, $15-18,20,25]$. Given the increasing interest in such applications, it is important to know whether or not the topological implications of the convexity of the upper and lower sections of $S$, valid when the range of $S$ is $R^{n}$, still hold when the range of $S$ is an arbitrary locally convex topological space. Propositions 1 and 2 in this note demonstrate that there is no hope of obtaining general results similar to Theorems 1 and 2, if $R^{n}$ is replaced with an infinite-dimensional space. One approach to deal with this unfortunate fact is to impose additional assumptions on $S^{-1}$. However, such assumptions often lack a clear economic interpretation, and they are more difficult to verify than simply assuming that $S$ has open and convex upper sections. It is important to keep in mind that the counter-examples of this note only show that a particular method (Theorems 1 and 2) fails to establish the existence of certain elements of interest (maximal elements, continuous selection, fixed points, Nash equilibria) in infinite dimensional settings. These counter-examples do not rule out the possibility that other methods might succeed. Therefore, in applications involving infinite-dimensional spaces, finding sufficient conditions for the existence of maximal elements, continuous selections, fixed points, and Nash equilibria that can be imposed on $S$ (rather than on $S^{-1}$ ), and that can be easily interpreted and verified, continues to be a topic worthy of further investigation.

\section{REFERENCES}

[1] J. C. Moore, "Mathematical Methods for Economic Theory, 2,” Studies in Economic Theory, Springer-Verlag, Berlin, Vol. 10, 1999.

[2] R. T. Rockafellar and R. J.-B. Wets, "Variational Analysis," Grundlehren der mathematischen Wissenchaften, Springer, Berlin, Vol. 317, 2009.

[3] N. Stokey, R. E. Lucas and E. Prescott, "Recursive Methods in Economic Dynamics," Harvard University Press, Cambridge, 1989.

[4] C. D. Aliprantis, G. Camera and F. Ruscitti, "Monetary Equilibrium and the Differentiability of the Value Function," Journal of Economic Dynamics and Control, Vol. 33, No. 2, 2009, pp. 454-462. doi:10.1016/j.jedc.2008.06.010

[5] L. M. Benveniste and J. A. Scheinkman, "On the Differentiability of the Value Function in Dynamic Models of Economics,” Econometrica, Vol. 47, No. 3, 1979, pp. 727-732. doi:10.2307/1910417

[6] T. Kim, "Differentiability of the Value Function: A New Characterization," Seoul Journal of Economics, Vol. 6, 1993, pp. 257-265.

[7] D. Gale and A. Mas-Collell, “An Equilibrium Existence 
Result Theorem for a General Model without Ordered Preference," Journal of Mathematical Economics, Vol. 2, 1975, pp. 9-15. doi:10.1016/0304-4068(75)90009-9

[8] D. Gale and A. Mas-Collell, "Corrections to an Equilibrium Existence Theorem for a General Model without Ordered Preferences," Journal of Mathematical Economics, Vol. 6, No. 3, 1979, pp. 297-298. doi:10.1016/0304-4068(79)90015-6

[9] E. Michael, “Continuous Selections I,” Annals of Mathematics, Vol. 64, No. 2, 1956, pp. 361-382. doi:10.2307/1969615

[10] G. Tian, "On the Existence of Equilibria in Generalized Games,” IJGM, Vol. 20, 1992, pp. 247-254.

[11] N. C. Yannelis and N. B. Prabhakar, "Existence of Maximal Elements and Equilibria in Linear Topological Spaces," Journal of Mathematical Economics, Vol. 12, 1983, pp. 233-245. doi:10.1016/0304-4068(83)90041-1

[12] N. Sun, "Bewley's Limiting Approach to Infinite Dimensional Economies with lsc Preferences,” Economics Letters, Vol. 92, No. 1, 2006, pp. 7-13. doi:10.1016/j.econlet.2006.01.006

[13] T. F. Bewley, "Existence of Equilibria in Economies with Infinitely Many Commodities," Journal of Economic Theory, Vol. 4, No. 3, 1972, pp. 514-540. doi:10.1016/0022-0531(72)90136-6

[14] G. Chichilnisky and P. Kalman, “An Application of Functional Analysis of Model of Optimal Allocation of Resources with an Infinite Horizon,” Journal of Optimization Theory and Applications, Vol. 30, No. 1, 1980, pp. 19-32. doi:10.1007/BF00934586

[15] G. Chichilnisky and Y. Zhou, "Smooth Infinite Economies,” Journal of Mathematical Economics, Vol. 29, 1998, pp. 27-42. doi:10.1016/S0304-4068(97)00009-8

[16] M. Florenzano, "On the Existence of Equilibria Space in Economies with an Infinite Dimensional Commodity Space," Journal of Mathematical Economics, Vol. 13,
1983, pp. 207-219. doi:10.1016/0304-4068(83)90039-3

[17] A. Mas-Collel and W. R. Zame, "Equilibrium in Infinite Dimensional Spaces,” In: W. Hildenbrand and H. Sonnenschein, Eds., The Handbook of Mathematical Economics, Elsevier, London, Chapter 34, Vol. 4, 1991.

[18] S. Toussaint, "On the Existence of Equilibria in Economies with Infinitely Many Commodities and without Ordered Preferences,” Journal of Economic Theory, Vol. 33, No. 1, 1984, pp. 98-115. doi:10.1016/0022-0531(84)90043-7

[19] N. C. Yannelis and M. Ali-Khan, "Equilibrium Theory in Infinite Dimensional Spaces,” Springer-Verlag, New York, 1991.

[20] W. Zame, "Competitive Equilibria Space in Production Economies with an Infinite Dimensional Commodity Space,” Econometrica, Vol. 33, 1987, pp. 1075-1108. doi:10.2307/1911262

[21] W. Rudin, "Functional Analysis," 2nd Edition, Interntional Series in Pure and Applied Mathematics, McGrawHill, New York, 1991.

[22] C. D. Aliprantis and K. C. Border, "Infinite Dimensional Analysis: A Hitchhiker’s Guide,” 3rd Edition, SpringerVerlag, Berlin, 2006.

[23] T. C. Bergstrom, R. P. Parks and T. Rader, "Preferences Which Have Open Graphs," Journal of Mathematical Economics, Vol. 3, 1976, pp. 265-268. doi:10.1016/0304-4068(76)90012-4

[24] J. Zhou, "On the Existence of Equilibrium for Abstract Economies,” Journal of Mathematical Analysis and Applications, Vol. 193, No. 3, 1995, pp. 839-858. doi:10.1006/jmaa.1995.1271

[25] C. D. Aliprantis and R. Tourky, "Equilibria in Incomplete Assets Economics with Infinite Dimensional Spot Markets," Economic Theory, Vol. 38, No. 2, 2008, pp. 221262. doi:10.1007/s00199-007-0247-2 